\title{
Granular impacts: how far the Boussinesq model can go?
}

\author{
Francisco Martinez $^{1^{*}}$ and Claudia Gonzalez \\ ${ }^{1}$ Escuela de Ingeniería Civil, Pontificia Universidad Católica de Valparaíso (PUCV), Chile
}

\begin{abstract}
A key problem on granular impacts deals with the determination of the mechanical response of the grains due to the impact of the intruder. This topic has been poorly addressed in the literature so far, a gap to which this study aims to contribute by measuring the pressure distribution at the bottom of a loose and dry sandy bed, impacted by a heavy sphere of fixed diameter. Exploring different bed thicknesses and intruder's dropping height, we have found that the structure of this distribution is very similar to the Boussinesq model, initially proposed for a static point-force acting over an isotropic-elastic medium. This surprising result opens up many challenging questions that could help validate or refute this model in other scenarios.
\end{abstract}

\section{Introduction}

Granular impacts have called the attention of many scientific areas in the last decades, such as geology, physics and soil mechanics. In this last one, granular impacts are typically involved on dynamical compaction processes performed to improve soil quality, its stability and bearing capacity $[1,2]$. On this way, granular impacts phenomenon has allowed to get insight about the collective friction properties of the grains, its rheological behaviour and the mechanical response issue from the application of an external load. Despite of this importance, very few studies have addressed this particular topic. Boussinesq solution was a pioneer attempt to describe the vertical stress response $P(r, z)$ in an inner point of a semi-infinite, homogeneous, isotropic and elastic medium under a single static pointforce at its top (see Fig.1), this is:

$$
P(r, z)=\frac{3 F}{2 \pi} \frac{1}{z^{2}}\left(\left(\frac{r}{z}\right)^{2}+1\right)^{-5 / 2}=\frac{F}{z^{2}} I_{B}(r, z)
$$

where $F$ is the surface concentrated point-force and $I_{B}(r, z)$ an influence coefficient dependent on the position $(r, z)$ into the soil $[1,2]$. Some authors have proposed the need of considering the grains elasticity into these equations, given the compressibility of the media. Kelvin [3] proposed the next formula to calculate the over pressure into an elastic, isotropic, homogeneous medium of Poisson ratio $(v)$, under a concentrated pointforce:

$$
P(r, z)=\frac{F}{8 \pi(1-v)} \frac{z}{\rho^{3}}\left(1-2 v+3\left(\frac{z}{\rho}\right)^{2}\right)
$$

where $\rho^{2}=r^{2}+z^{2}$. Inspired on Eq.1, Westergaard proposed a law with direct applications on soil mechanics that includes the parameter $v$ [2], this is:

$$
P(r, z)=\frac{F}{2 \pi z^{2}} \frac{\sqrt{\frac{2(1-2 v)}{(1-v)}}}{\left(\frac{(1-2 v)}{(2-v)}+\left(\frac{r}{z}\right)^{2}\right)^{3 / 2}}
$$

Some studies have measured this response at the bottom of a granular pile under the action of a static

\footnotetext{
* Corresponding author: francisco.martinez@pucv.cl
}

A video is available at https://doi.org/10.48448/6xkd-4379 point-force applied at the top of the pile [4-6]. Although the characteristics of the material turns this scenario far from Boussinesq original hypothesis, this response has surprisingly shown to be well represented by the Eq. 1 . On the other side, the use of frictional grains has made possible to extend the validity of Eq. 1 for larger forces, reducing the effects of mechanical anisotropy of the pile [7].

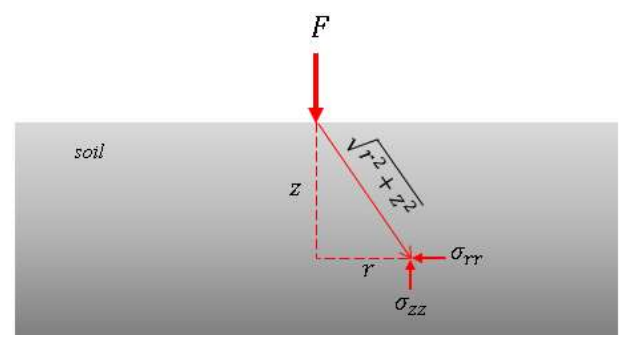

Fig. 1. Sketch of a single point-force applied at the top of a granular bed. We define the reference system and the vertical stress $\boldsymbol{\sigma}_{\mathbf{z z}}=\boldsymbol{P}(\boldsymbol{r}, \boldsymbol{z})$, according to Eq. 1 .

In addition, a single peak of pressure beneath the point-force has been obtained for hard-spheres piles in opposition to softer materials, which can show up to two pressure peaks near the middle of the pile [5]. All these results have given new insight about the mechanical response of granular piles under external forces, but at the same time most of them have dealt with static forces acting on the top of granular piles. In this context, the aim of the present study is to explore to which to extent the Boussinesq law can be applied when a dry and loose granular pile is hit by an intruder falling from the rest.

\section{Experimental set-up}

\subsection{Materials}

In our experiments we used dry and poorly graded sand (SP), whose physical parameters are shown in the Table 1 . The material was deposited by pluviation in an acrylic square cross-section deposit of of $265 \times 265 \mathrm{~mm}, 10 \mathrm{~mm}$ of bottom thickness, $5 \mathrm{~mm}$ wall-thickness. The granular pile's thickness $h$ varies in the range $90-190 \mathrm{~mm}$ (see Fig. 2). The intruder is a steel sphere of diameter $D=60$ $\mathrm{mm}$, density $\rho=7.9 \mathrm{~g} / \mathrm{cm}^{3}$ and mass $M=0.886 \mathrm{~kg}$. 
The intruder's dropping system consists of a mechanical brake manually activated so that the sphere is released with zero initial speed. A rigid vertical structure supports the tank and the brake, allowing to test the dropping heights $H=0.50,0.75$ and $1.00 \mathrm{~m}$ (measured from the center of the sphere to the surface of the pile).
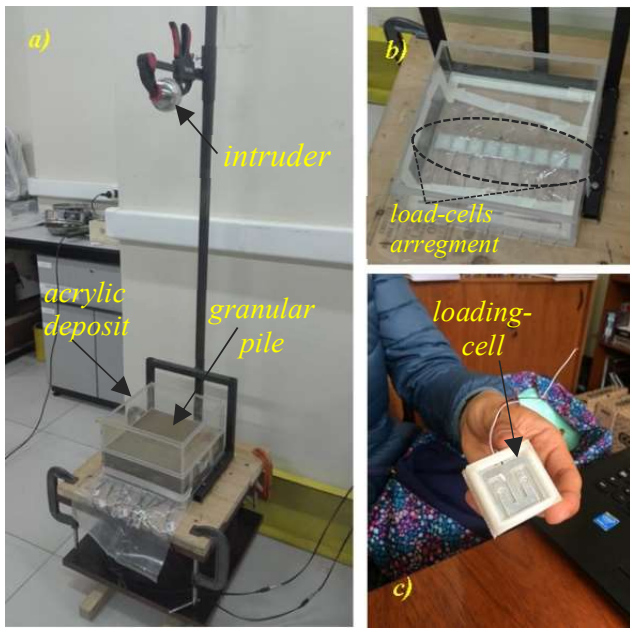

Fig. 2. a) General view of the experimental set-up; b) the collection of the loading-cells at the bottom of the acrylic box; c) a closer view of one of the load-cell used in the experiments.

Table 1. Grain size parameters.

\begin{tabular}{|c|c|c|}
\hline Parameter & Value & Units \\
\hline $\mathrm{d}_{10}{ }^{(1)}$ & 0.115 & {$[\mathrm{~mm}]$} \\
\hline $\mathrm{d}_{30}{ }^{(1)}$ & 0.170 & {$[\mathrm{~mm}]$} \\
\hline $\mathrm{d}_{60}{ }^{(1)}$ & 0.223 & {$[\mathrm{~mm}]$} \\
\hline $\mathrm{C}_{\mathrm{u}}(2)$ & 1.194 & - \\
\hline
\end{tabular}

(1) $d_{n}=$ diameters for which the percentage $n$ of the particles are smaller than this value. ${ }^{(2)} \mathrm{C}_{\mathrm{u}}=$ coefficient of uniformity $=$ $\mathrm{d}_{60} / \mathrm{d}_{10}$.

To prevent any inclination effect (see e.g. [6]), we have used an Automatic Level Surveying Instrument (Spectra Precision AL32M), assuring the verticality of the brake and the centring of the impact point. Finally, a collection of nine loading-cells (model TAL 106, HT sensor Technology Co.) locates at the bottom of the tank. The cells are uniformly distributed along the bottom and covered with a skinny polyethylene layer to prevent any contact with the grains. The surface contact area of each cell is $S=26 \times 26 \mathrm{~mm}^{2}$. The nominal capacity of each cell ranges from 29 [N] (near sides) up to 50 [N] (at $\mathrm{r}=0$ ). Additionally, an electronic acquisition system records the time force signal emitted by each cell at a sampling rate of $2.5[\mathrm{kS} / \mathrm{s}]$. This sampling rate is in agreement with the typical time values found in literature $[8,9]$.

\subsection{Methodology}

The sand is gently poured into the tank by a rainlike method proposed by [10], filling the tank up to the thickness $h$. Later, the granular surface is gently smoothed with a ruler until obtain a plane surface. The preparation method allows to get a reproducible deposit, whose bulk density $\left(\bar{\rho}_{s}\right)$ falls in the range 1.43-1.54 $\mathrm{g} / \mathrm{cm}^{3}\left(1.47 \mathrm{~g} / \mathrm{cm}^{3}\right.$ in average). The tank is not deeper enough to observe a Janssen-like effect, then the granular pressure prior to the impact can be approximated by $\bar{\rho}_{s} g h$. This term falls in the range 1.46$3.08[\mathrm{kPa}]$, much lower than the nominal capacity of the sensors. This value is the offset of the cells. Hereinafter, all the results will correspond to the pressure over this offset in coherence with Eq. 1. Once the cells are ready to register, the sphere is carefully dropped from a height $H$. Each load cell registers a force time signal $F(r, t)$, transmitted to a computer with an arduino-based system. Finally, for each $H$ a group of nine force time series at the bottom of the pile was obtained, in the range [-114, $114 \mathrm{~mm}$ ]. The vertical mean pressure at each cell is given by $P(r)=\frac{F_{\max }(r)}{S}$, where $F_{\max }(r)$ is the peak value registered by each cell and $S$ is its contact area with the grains. Once the experiment is finished, we replace all the grains; we take away the pile sand, clean the acrylic box and fill it again by following the same preparation method. We repeat this for different bed thickness and dropping heights, recording the distribution of pressure every time.

\section{Results}

\subsection{Pressure distribution at the bottom}

We only have focused on the peak force generated by the impacts, as shown in the Figures $3 \mathrm{a}$ and $3 \mathrm{~b}$. In the Figure $3 \mathrm{c}$ we can observe these peaks after five repetitions of the experiment, under the same initial conditions, near the middle of the pile and close to the sidewalls. There is an expected variability of these values beneath the impact point, maybe due to large rearrangements of grains during the hit; but this variability decreases significantly when we get closer to the walls of the tank.
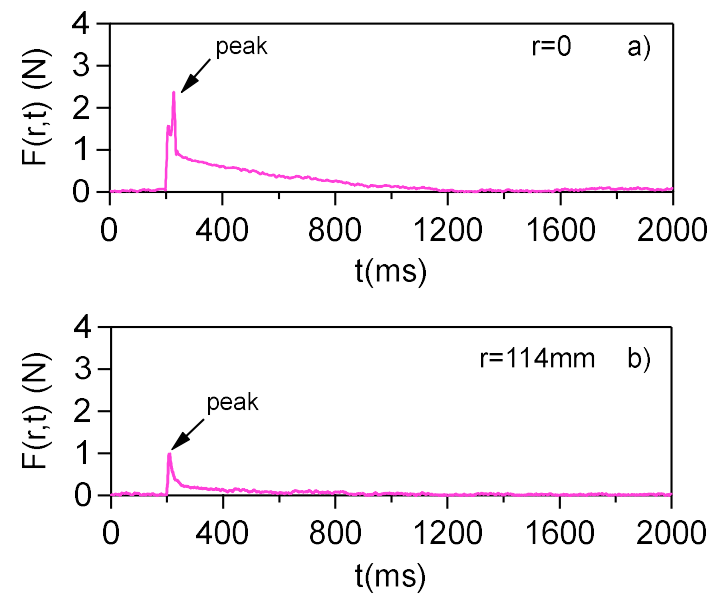


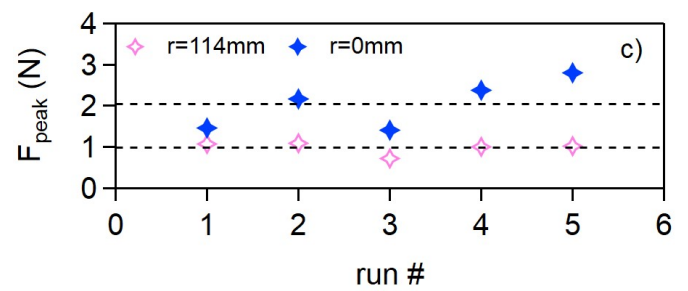

Fig. 3. Force signal at a) $r=0$ and b) $r=114.0 \mathrm{~mm}$. c) The force peak values for both positions; the dashed lines are the averages of each set, these are $2.05 \pm 0.27$ and $0.98 \pm 0.07$, respectively.

In the Figure 4 we show the dimensionless pressure $P(r) / P(0)$ versus $r / h$ for all the experiments, with $P(0)$ the pressure at the middle of the pile. The data seems to collapse around a well-defined curve, although with significant scattering. This collapse proposes a scaling of the type $P(r) / P(0) \propto f(r / h)$, where $f$ is an unknown fitting function. In the same figure, we have included the fits obtained from Eqs. 1, 2, 3 and the Lorentz function $\left(1+(r / h)^{2}\right)^{-1}$.
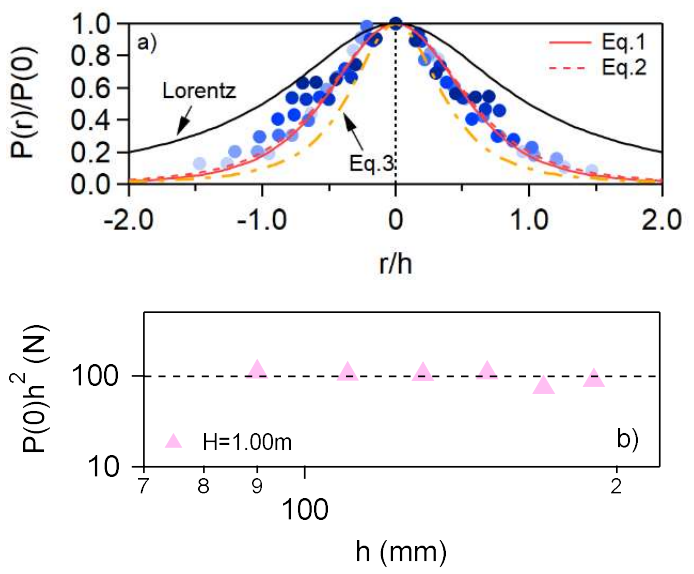

Fig. 4. Some results for $\boldsymbol{H}=1 \mathrm{~m}$. a) Pressure curves scaled by $\boldsymbol{P}(\mathbf{0})$ vs. $\boldsymbol{r} / \boldsymbol{h}$. The graph includes the Lorentz model (black line), the Westergaard solution (dashed-point line). Kelvin (red dashed line) and Boussinesq solution (red line), were obtained for $\boldsymbol{v}=0.27$. b) We observe the scaled curve $\boldsymbol{P}(\mathbf{0}) \boldsymbol{h}^{\mathbf{2}}$ vs. $\boldsymbol{h}$. All data was obtained from [11].

The Figure 4a shows Eq. 2 for $v=0.27$. This seems to be a characteristic fit value in the literature, although some studies have discussed about the lack of sensitiveness of this equation respect to this parameter [6]. On the other side, the fits obtained from Eq.1 and Eq. 2 looks very similar, then we are not committing a significant error considering the Boussinesq model to predict the inner stress in our range. The normalized pressure is also sharper than a Lorentzian profile, similar as reported by [4], but the Westergaard solution underestimate our results. The Figure $4 \mathrm{~b}$ suggests that $P(0) h^{2} \propto K$, with $K$ like a constant of force. This constant takes the values $46.1 \pm 2.3 \mathrm{~N}, 68.5 \pm 2.3 \mathrm{~N}$ and $88.8 \pm 2.0 \mathrm{~N}$ for $H=0.5,0.75$ and $1 \mathrm{~m}$ respectively. If this scaling is correct, we should obtain a decreasing behaviour between $P(0) / K$ and $h$. Figure 5 compares both parameters for different drop heights, showing the decreasing law as expected.

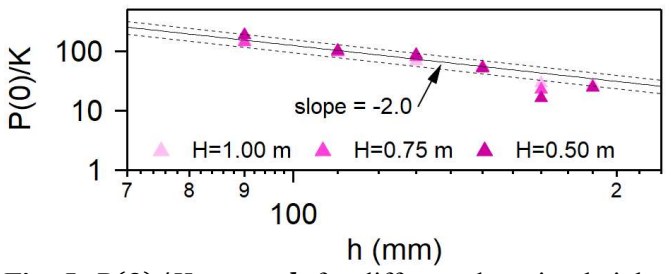

Fig. 5. $\boldsymbol{P}(\mathbf{0}) / \boldsymbol{K}$ versus $\boldsymbol{h}$ for different dropping heights. The continuous line is a power law fit of negative slope (-2); the point-lines defines a $\pm 25 \%$ scattering band $(72.2 \%$ of data falls within).

The variation in $H$ is negligible, so the measures in the Figure 5 can be fit by a power law fit of mean slope -2 . There is a little difference between Eq. 1 and 2 and given the extended use of the Boussinesq model in engineering practice. From Figs. $4 \mathrm{a}, 4 \mathrm{~b}$ and 5 we can propose the next global scaling:

$$
P(r, h)=\frac{K}{h^{2}} I_{B}(r, h)
$$

Then, the question to answer here is what is the suitable model for the force being applied at the top of the pile for granular impact?

\subsection{A model for the surface point-force}

To get insight about this force we propose a very simple model taking into account a static contribution (equals to the weight of the intruder $\mathrm{Mg}$ ) and a dynamic term arising from the momentum exchange between the intruder and the grains at the impact, $\frac{1}{2} \rho A u^{2} . M$ is the mass of the sphere, $A=\pi D^{2} / 4$ its projected area over the surface, $g$ is the gravity acceleration and $u \approx \sqrt{2 g H}$ the impact velocity. The static and dynamic contribution lead to the next model for the concentrated point-force:

$$
F=M g+\frac{1}{2} \rho A u^{2}
$$

with $\rho$ the density of the sphere. By evaluating Eq.5, in our experiments we have found that $K=\lambda F$ with $\lambda=0.40 \approx 3 / 2 \pi$. From this last equality, we can define the next dimensionless number:

$$
\zeta=\frac{P h^{2}}{F I_{B}}
$$

If Eq. 5 is correct, the upper limit of $\zeta$ should be 1. In the Figure 6 a we compare $\zeta$ vs. $r / h$, for all the experiments. Although the scattering is significant, our data reasonable collapses into a curve that can be fit by a horizontal line, whose mean value is $0.99 \pm 0.05$. This fit works reasonably well in the range $r / h<1$; however, for $r / h>1$ the number $\zeta$ shows a deviation from this average (indicated by the dashed vertical line), suggesting possible boundary effects on this scaling. 

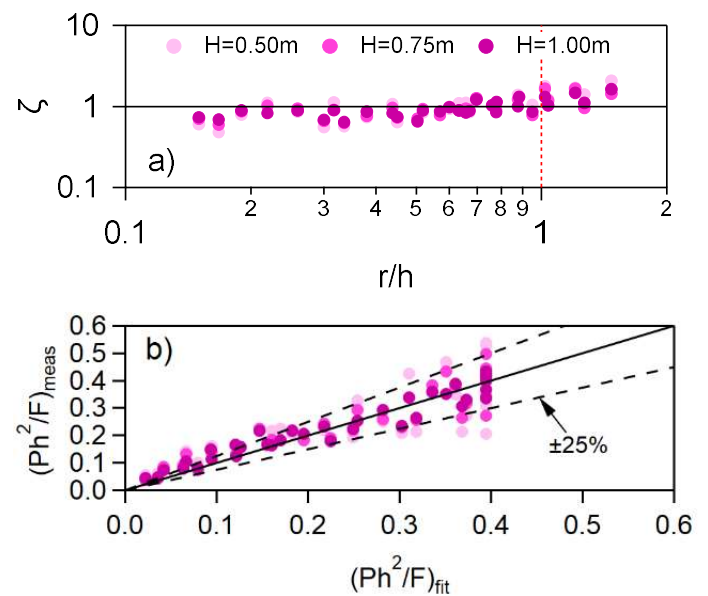

Fig. 6. a) $\boldsymbol{\zeta}$ versus $\boldsymbol{r} / \boldsymbol{h}$ for different dropping heights. The continuous line is a horizontal fit, whose average is close to 1 . The dashed vertical line just splits the graph in the region $\boldsymbol{r} / \boldsymbol{h}>1$. In b) we show the estimation of the goodness of fit of Eq. 1. The continuous line is a linear fit of slope 1 . The dashed lines represent the boundaries of a $\pm 25 \%$ scattering band.

In the Figure $6 \mathrm{~b}$ we have drawn the scattering band related to the fit proposed by Eqs. 4 and 5. Almost 74\% of measures fall into a $\pm 25 \%$ band, an acceptable result considering that we are dealing with a violent phenomenon in natural grains. Then, we can finally propose the next overall scaling for our experimental data:

$$
P(r, h) \approx \frac{3}{2 \pi} \frac{F}{h^{2}} I_{B}(r, h)
$$

\section{Conclusion and Discussion}

In the present article we have determined the mechanical response of a sandy bed hit by a heavy sphere drop from the rest. In a limited range, we show that is possible to extend the Boussinesq model for granular impacts. This result is supported on a point-force model acting at the surface, which consider the weight of the intruder and the momentum exchange with the grains. The fact of considering the elasticity of grains through the Poisson ratio, does not change this result. The Kelvin solution for an elastic isotropic medium also fit well our data, with $v<0.5$, with little sensitiveness to this parameter. The use of other models (typically found in engineering practice), like Westergaard's equation, underpredict our results. In consequence, Boussinesq model reveals to be a robust law to be used in a dynamical scenario.

This striking result lets, however, some important open questions needing further research. Firstly, it is not clear to which to extent the relation between the time scales involved during the impact and the granular properties can influence the magnitude of these bottom pressure distributions. Some authors have pointed that the traveling time of the sound waves due to impact is a key parameter to understand the propagation of force chains through the grains. The topology of these force chains is the key to understand the pressure response in deeper layers of a granular pile [12]. On the other side, we wonder about how this response could change under high-impact scenarios. Once again, the stiffness, shape and surface texture of grains should play a substantial role on this propagation dynamics and then, the expected force peak values at the bottom. All these important challenging questions will be faced in future works on the topic.

We acknowledge the PUCV's Vicerrectoría de Investigación for the grant Investigador Emergente 039.371/19. We also thank Hugo Tapia (PUCV) and Daniel Yunge (PUCV) for their technical support on the construction of the set-up.

\section{References}

1. J.E. Bowles. Foundation Analysis and Design (McGraw-Hill International, 1996)

2. V.N.S. Murthy, Geotechnical Engineering: Principles and Practices of Soil Mechanics and Foundation Engineering (CRC Press, 2002)

3. M.L. Kachanov, B. Shafiro, I. Tsukrov. Handbook of Elasticity Solutions (Springer Science \& Business Media, 2013)

4. G. Reydellet, E. Clement, Phys. Rev. Lett. 86, 3308 (2001)

5. J.P. Bouchaud, P. Claudin, E. Clement, M. Otto and G. Reydellet. C R Phys 3(2), 141 (2002)

6. A.P.F. Atman, P. Brunet, J. Geng, G. Reydellet, G. Combe, P. Claudin, R.P. Behringer, E. Clement, J. Phys. Condens. Matter 17(24) (2005)

7. C. Goldenberg, I. Goldhirsch, Phys. Rev. E 77, 041303 (2008)

8. S. Ji, X. Chen, P. Li, Y. Yan, Granular Matter: A Special Buffer for Impact Load, in AIP Conference Proceedings 1542(1), 401 (2013)

9. S. Takizawa, H. Niiyaa, T. Tanabe, H. Nishimori, H. Katsuragi. Physica D, 386-387, 8-13(2019)

10. C.M. González, M.P. Romo, Serie Azul Instituto de Ingeniería. Universidad Nacional Autónoma de México SID 691(2015)

11. F. Martínez, M.P. Urrea, C.M. González, G. Varas. Granul. Matter 23(3) (2020).

12. T. Takahashi, A. H. Clark, T. Majmudar, L. Kondic. Phys. Rev. E 97, 012906 (2018) 